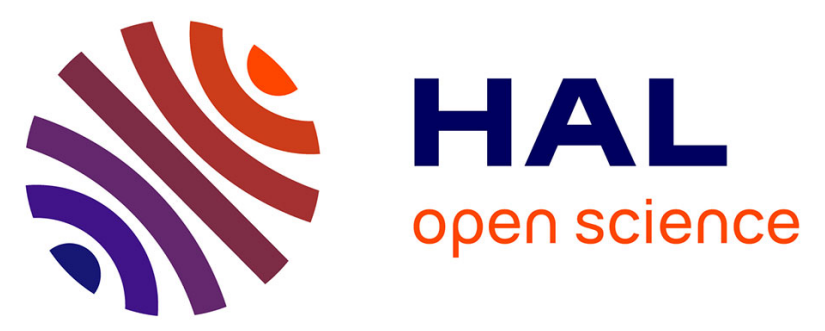

\title{
Field-periodic magnetoresistance oscillations in thin graphite single crystals with columnar defects
}

\author{
Yuri I. Latyshev, A. Yu Latyshev, Andrei P. Orlov, A. A. Chekin, V. A.
}

Bykov, Pierre Monceau, Cornelis Jacominus van Der Beek, Marcin

Konczykowski, Isabelle Monnet

\section{To cite this version:}

Yuri I. Latyshev, A. Yu Latyshev, Andrei P. Orlov, A. A. Chekin, V. A. Bykov, et al.. Field-periodic magnetoresistance oscillations in thin graphite single crystals with columnar defects. JETP Letters, 2009, 90 (6), pp.480-484. 10.1134/S0021364009180167. hal-00419331

\section{HAL Id: hal-00419331 \\ https://hal.science/hal-00419331}

Submitted on 23 Sep 2009

HAL is a multi-disciplinary open access archive for the deposit and dissemination of scientific research documents, whether they are published or not. The documents may come from teaching and research institutions in France or abroad, or from public or private research centers.
L'archive ouverte pluridisciplinaire HAL, est destinée au dépôt et à la diffusion de documents scientifiques de niveau recherche, publiés ou non, émanant des établissements d'enseignement et de recherche français ou étrangers, des laboratoires publics ou privés. 


\section{Периодические по полю осцилляции магнетосопротивления тонких монокристаллов графита с колоннообразными дефектами}

Ю.И.Латышев ${ }^{1}$, А.Ю. Латышев ${ }^{1}$, А.П. Орлов ${ }^{1}$, А.А. Щекин ${ }^{2}$, В.А. Быков ${ }^{2}$, П. Монсо ${ }^{3,4}$, К. ван дер Бек ${ }^{5}$, М. Конциковский ${ }^{5}$, И. Монне

${ }^{1}$ Институт радиотехники и электроники им. В.А. Котельникова РАН, Моховая 11-7, 125009 Москва, Россия.

23АО “НТ МДТ”, Зеленоград, корп. 317-А, а/я 158, 124482 Москва, Россия.

${ }^{3}$ Институт Нееля, 38042 Гренобль, Франция.

${ }^{4}$ Лаборатория сильных магнитных полей, 38042 Гренобль, Франция.

${ }^{5}$ Лаборатория иррадиации твердых тел, Политехнический институт, 91128 Палезо, Франция.

${ }^{6}$ Центр исследований взаимодействия ионов с материей и фотоники (CIMAP, GANIL) 14050 Кан, Франция.

e-mail: yurilatyshev@yahoo.com

PACS: 71.25.+a, 73.43.Qt, 73.63.-b

Исследовано магнетосопротивление тонких монокристаллов графита с колоннообразными дефектами (КД). Обнаружен периодический по полю вклад в магнетосопротивление с периодом 7,5 Тл. Оценка диаметра КД с помощью атомносилового микроскопа показывает, что периодичность осциллирующего вклада по потоку близка hc/e на дефект. Результат согласуется с измерениями осцилляций магнетосопротивления Ааронова-Бома на графеновых мезоскопических кольцах [S. Russo et al. Phys. Rev. B77, 085413 (2008)]. 
Открытие графена с бесмассовыми носителями, дираковскими фермионами [1], возродило интерес к исследованиям графита и, особенно, тонких монокристаллов графита толщиной 50-100 нм. На таких кристаллах был установлен значительный вклад в поглощение ИК диапазона на переходах между уровнями Ландау с энергиями, характерными для дираковских фермионов [2]. Наличие дираковских фермионов в графите было также подтверждено с помощью STM [3] и межслоевого туннелирования [4] в сильных магнитных полях.

Другим примечательным свойством дираковских фермионов является отсутствие рассеяния назад $[5,6]$ и связанное с этим отсутствие эффектов слабой локализации. Недавние исследования эффекта Ааронова-Бома на мезоскопических кольцах графена с диаметром $\approx 700$ нм выявили наличие слабых осцилляций магнетосопротивления с периодом по потоку в кольце $h c / e$, тогда как вклад $h c / 2 e$, связанный со слабой локализацией, был сильно подавлен [7].

В данной работе обнаружен эффект типа эффекта Аронова-Бома в магнетосопротивлении тонких монокристаллов графита с искусственными дефектами малых размеров 10-20 нм. Для этого тонкие кристаллы графита облучались тяжелыми ионами с высокой энергией, соответствующей образованию колоннообразных дефектов. В этих условиях пролет иона оставляет в кристалле трек аморфного вещества глубиной в несколько микрон и однородным диаметром масштаба 10 нм. Аморфное вещество в коре колоннообразного дефекта обычно непроводящее или плохо проводящее, т.е. для носителей тока колоннообразный дефект представляет собой наноотверстие. Поскольку каждый ион создает идентичный колоннообразный дефект, облученный тонкий монокристалл можно рассматривать как ансамбль наноотверстий одинакового диаметра.

Для исследований мы использовали монокристаллы естественного графита высокого качества. Проводимость в плоскости слоев составляла при комнатной температуре $30-50$ мкОм см, а анизотропия проводимости вдоль и поперек слоев составляла $410^{3}$. При гелиевых температурах она достигала $310^{4}$. Высокое качество кристаллов было подтверждено также исследованиями на них магнетопропускания в ИК диапазоне [8] и осцилляций магнетосопротивления Шубникова- де Газа [9].

Тонкие монокристаллические слои толщиной 30-70 нм отслаивались от основного кристалла с помощью адгезионной ленты с последующим растворением ее клеевой основы в ацетоне. Затем они подхватывались из растворителя на подложки из сапфира. С помощью селективного травления в кислородном разряде из них формировалась полоска шириной 30-40 мкм и длиной около 200-400 мкм. Низкоомные электрические контакты осуществлялись холодной пайкой (прижимом) индием. Облучение тяжелыми ионами Хе с энергией 90 МэВ проводилось на линейном ускорителе в Кане (Caen), Франция. Дозы облучения варьировались от $10^{9}$ до $10^{10}$ дефектов/см². Направление пучка ионов было параллельно оси $c$ облучаемых кристаллов. Измерения магнетосопротивления образцов в полях до 32 Тл проводились спустя две недели после их облучения на биттеровском магните в лаборатории сильных магнитных полей (LCMI) в Гренобле. Измерения зависимостей сопротивления R от магнитного поля Н проводились 4-х зондовым методом на малом постоянном токе с помощью нановольтметра и программируемого источника тока. Магнитное поле изменялось линейно по времени со скоростью 5-60 мТл/сек.

На рис. 1 показана зависимость $\mathrm{R}(\mathrm{H})$ образца №3 с минимальной дозой облучения $10^{9}$ деф./см ${ }^{2}$ от магнитного поля, ориентированного вдоль оси дефектов, Н//c, при нескольких фиксированных температурах. В области до 8 Тл хорошо видны осцилляции Шубниковаде Гааза, а в области сильных полей, больших 10 Тл, видны три осцилляции с минимумами при 15, 22,5 и 30 Тл. Период этих осцилляций соответствует 7,5 Тл. Минимум при 7,5 Тл также прослеживается, но значительно слабее. Зависимости R(H) практически совпадали при развертке поля вверх и вниз, а также при изменении ориентации поля на противоположную, $\mathrm{H} \rightarrow-\mathrm{H}$. На рисунке показаны зависимости $\mathrm{R}(\mathrm{H})$ при 1,$2 ; 4,2 ; 10 ; 20$ и 32К. Видно, что амплитуда осцилляций уменьшается с ростом 
температуры, и они практически исчезают при температурах в области 10 - 20 К. В этой же температурной области исчезают и осцилляции Шубникова-де Гааза.

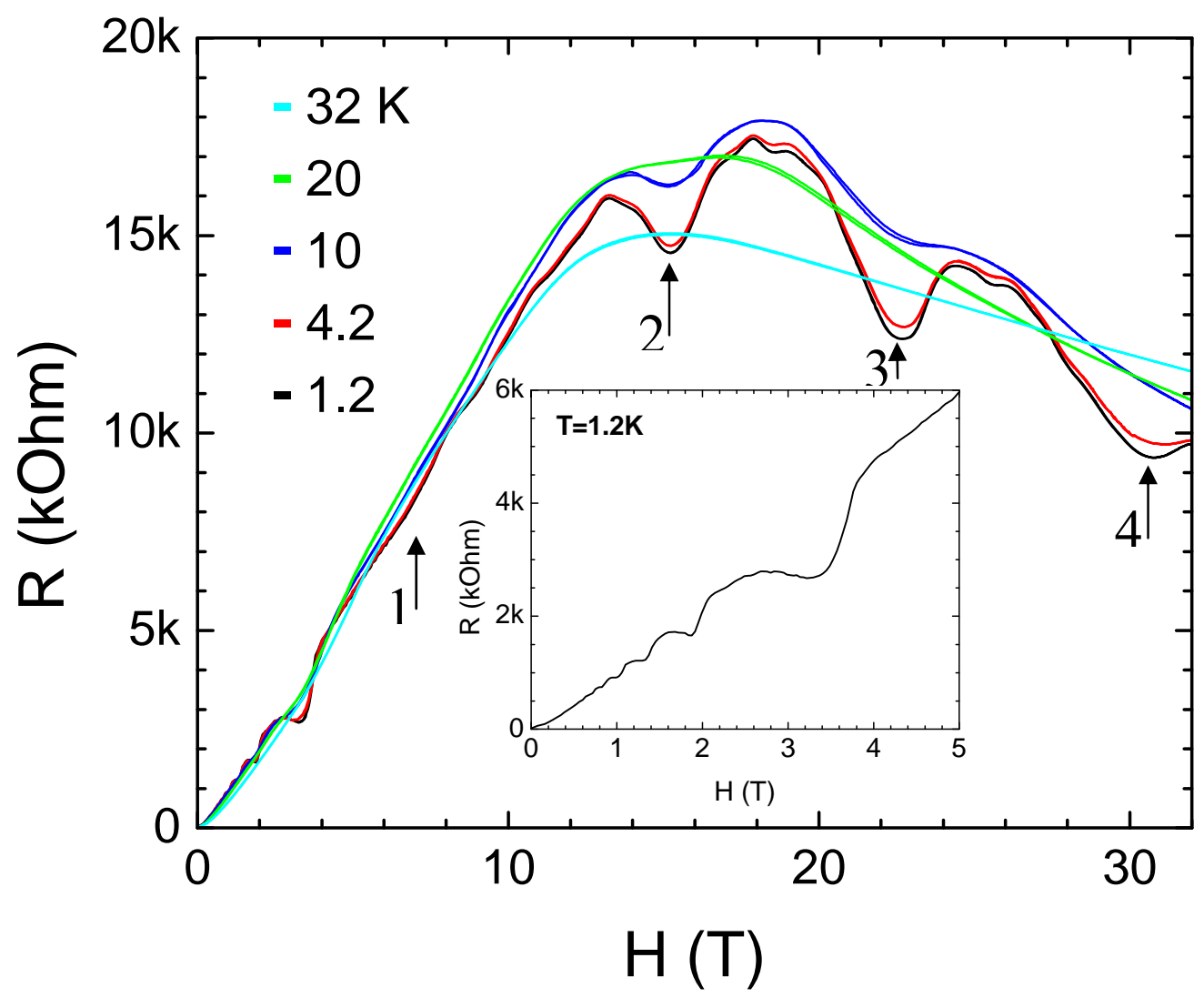

Рис. 1. Зависимость электросопротивления монокристалла графита №3 в плоскости $a b$ от магнитного поля $\mathrm{H} / / c$ при температурах $1.2,4.2,10,20,32 \mathrm{~K}$. Толщина образца 50 нм, концентрация колоннообразных дефектов $\mathrm{c}=10^{9}$ деф/см². Стрелками отмечена группа минимумов $\mathrm{R}\left(\mathrm{H}_{\mathrm{n}}\right)$, отвечающая закономерности $\mathrm{H}_{\mathrm{n}}=\mathrm{nH}_{0}$, где $\mathrm{n}$ - целое число, $\mathrm{H}_{0}=7.5$ Тл. На вставке увеличен фрагмент зависимости $\mathrm{R}(\mathrm{H})$ до 5 Тл при $\mathrm{T}=1.2 \mathrm{~K}$, иллюстрирующий осцилляции магнетосопротивления Шубникова-де Гааза.

Подобные крупномасштабные, периодические по полю осцилляции наблюдались и для двух других образцов с дозами облучения $310^{9}$ и $10^{10}$ деф./ $\mathrm{cm}^{2}$, но с меньшей амплитудой. Измерения необлученных образцов показывают отсутствие подобных осцилляций (рис.2). Вместе с тем, некая мелкая структура $\mathrm{R}(\mathrm{H})$ наблюдалась, как для облученных, так и для необлученных образцов (рис. 1,2).

В среднем зависимости $\mathrm{R}(\mathrm{H})$ необлученных образцов имеют вид, аналогичный объемным образцам монокристаллов графита высокого качества $[10,11]$ : они достигают широкого максимума в области полей 20 Тл, затем медленно спадают и имеют минимум при 28-30 Тл. В области максимума магнетосопротивление $\mathrm{R}\left(\mathrm{H}_{\max }\right) / \mathrm{R}(0)$ достигает $510^{3}$.

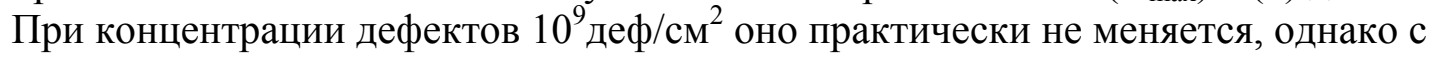
дальнейшим ростом концентрации дефектов магнетосопротивление при $\mathrm{H}=15-20$ Тл уменьшается (при с $=10^{10}$ деф./см² в 10 раз) и зависимости $\mathrm{R}(\mathrm{H})$ приобретают монотонный характер с насыщением при $\mathrm{H}>20$ Тл. 


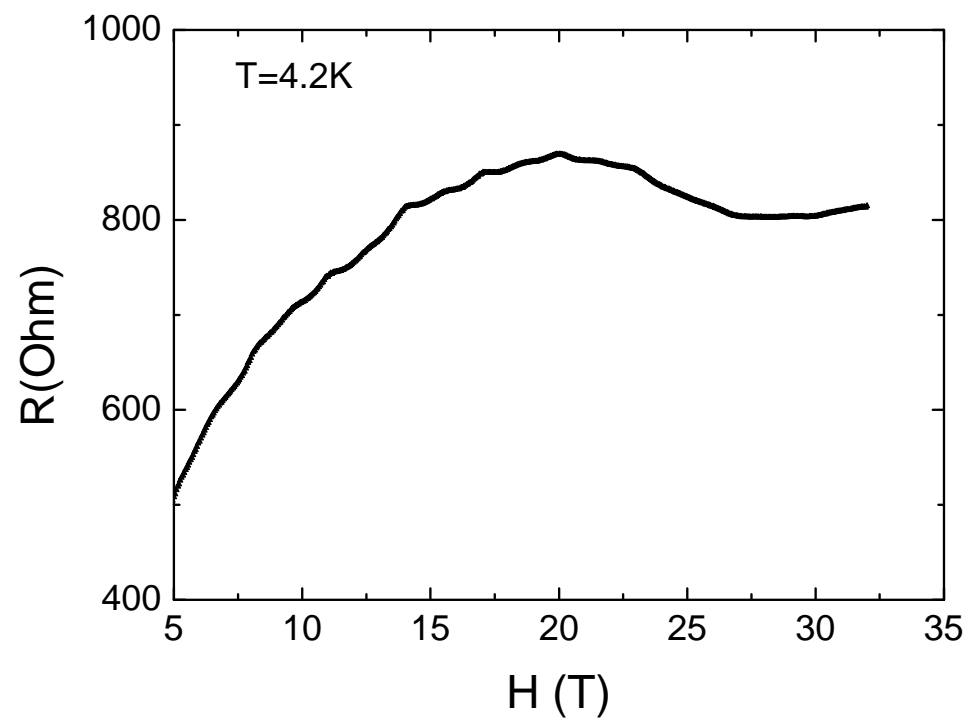

Рис. 2. Зависимость электросопротивления в плоскости $a b$ от магнитного поля $\mathrm{H} / / \mathrm{c}$ контрольного образца графита №4, толщиной 70 нм, без колоннообразных дефектов.

Анализ поверхности облученных образцов с помощью сканирующего атомно-силового микроскопа позволил идентифицировать колоннообразные дефекты по бугоркам (hillocks) выдавленного из них аморфного вещества [12] (рис. 3). Этот эффект известен и связан с релаксацией силовых напряжений, возникающих при формировании колоннообразного дефекта. Концентрация бугорков соответствует дозе облучения. Оценка диаметра КД D по плоской части бугорка дает величину $\mathrm{D} \leq 24$ нм при его высоте $\approx 6$ нм. В настоящее время мы не можем точнее определить диаметр КД.

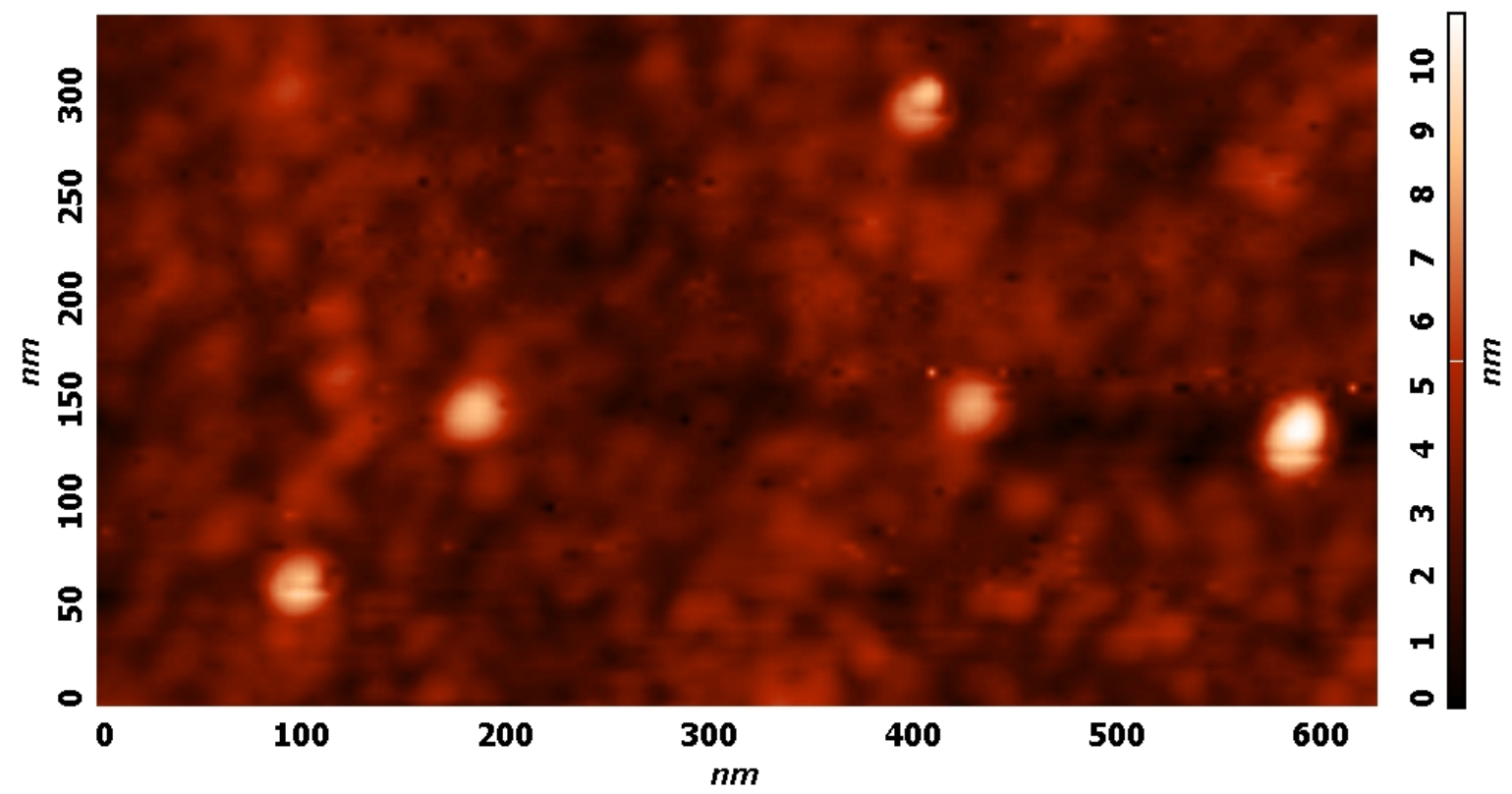




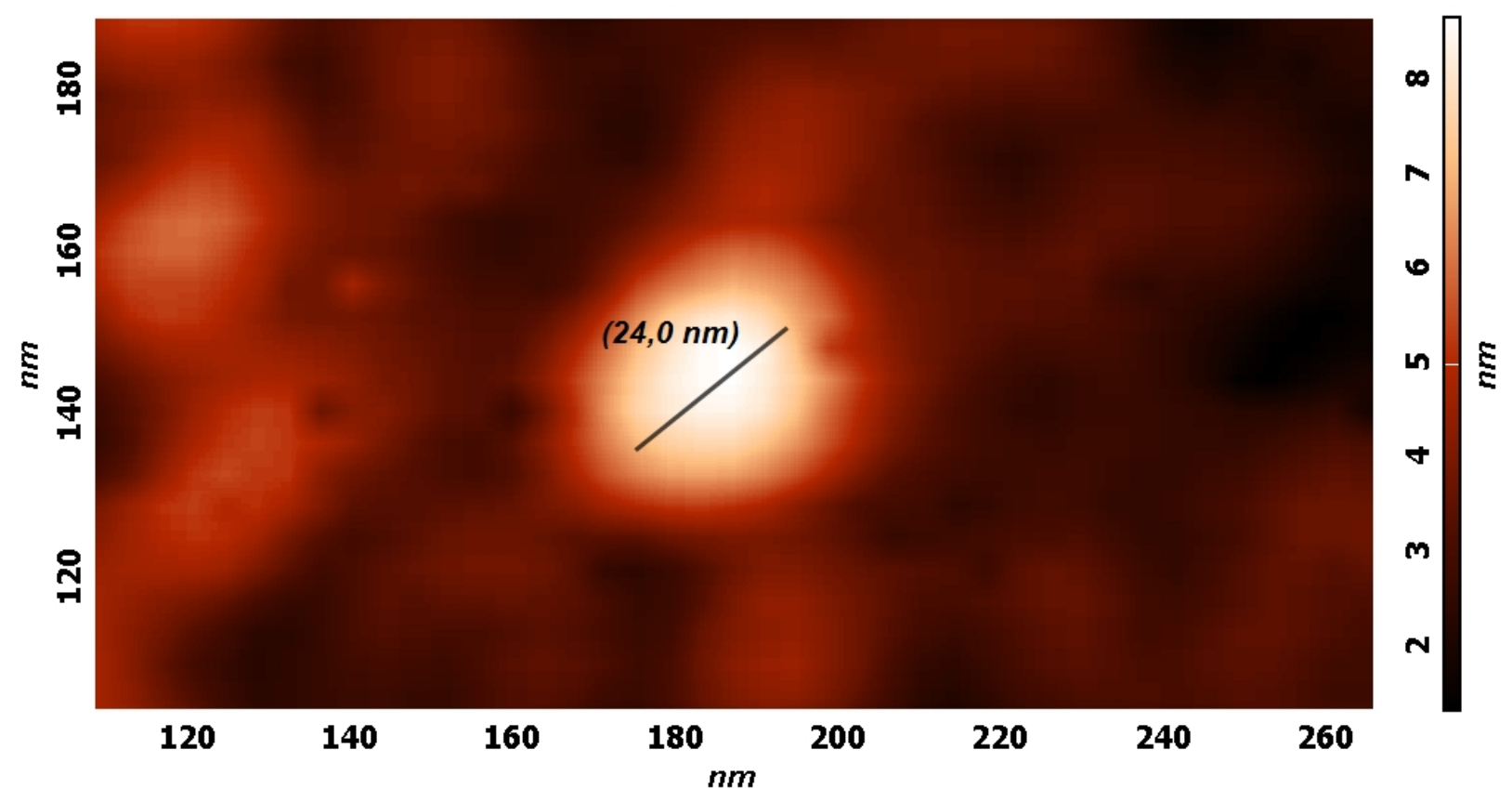

Рис. 3. Изображение группы (а) и единичного колоннообразного дефекта (б) на образце №3 в сканирующем атомно-силовом микроскопе (AFM). Колоннообразные дефекты воспринимаются на AFM, как бугорки выдавленного из них аморфного вещества (см. текст).

Перейдем теперь к обсуждению результатов. Аморфное вещество внутри колоннообразного дефекта обычно является непроводящим, и колоннообразный дефект рассматривают для носителей как полый цилиндр, пронизывающий всю толщу тонкого образца. В магнитном поле КД представляет собой наномагнит, двигаясь вокруг которого носители могут набирать фазу. Поскольку все КД имеют одинаковую площадь, все они пронизаны одинаковым магнитным потоком, поэтому носители, двигающиеся вокруг дефектов, набирают одинаковую дополнительную фазу. Например, в условиях, когда каждый дефект содержит квант потока разные носители, каждый, двигаясь вокруг своего КД, набирают при полном обороте одинаковую дополнительную фазу $2 \pi$. Это и определяет период осциллирующей части магнетосопротивления.

Экспериментальные данные соответствуют периоду по полю $\Delta \mathrm{H}=7,5$ Тл. В пересчете на магнитный поток и в предположении, что основной вклад дают траектории, близкие к периметру КД получаем $\Delta \Phi=\Delta \mathrm{H} \pi \mathrm{D}^{2} / 4=3,4 \pm 0,810^{-8}$ мТл см ${ }^{2}$, что, несмотря на большую неопределенность в размере КД, ближе к величине hc/e $\left(4,1410^{-8}\right.$ мТл см²), чем hc/2e $(2,07$ $10^{-8}$ мТл см ${ }^{2}$ ). Нужно также учесть, что из-за того, что длина $L_{H}$, определяющая радиус циклотронных орбит носителей $L_{H}=\sqrt{\hbar c /(e H)} \quad\left(L_{H}=8-4.6\right.$ нм в области полей 10-30Тл), меньше радиуса КД, эффективный диаметр орбиты носителей вокруг КД больше его геометрического диаметра. Учет этого обстоятельства сдвинет оценку $\Delta \Phi$ в сторону hc/e.

Полученные результаты согласуются с экспериментами на графеновых мезокольцах [7]. В [7] при внутреннем диаметре кольца 700 нм период осцилляций составлял 7 мТл, тогда как в нашем эксперименте при диаметре КД в 30 раз меньшем период осцилляций, пропорциональный $1 / \mathrm{D}^{2}$, примерно на три порядка больше - 7,5 Тл. Полученные 
результаты указывают на то, что в эксперименте, по-видимому, проявляется интерференция дираковских фермионов. На это указывает также то, что периодическими по полю оказываются минимумы осцилляций магнетосопротивления $\mathrm{H}_{\min }=\mathrm{n} \mathrm{H}_{0}$, при $\mathrm{n}=$ $2,3,4 ; \mathrm{H}_{0}=7.5$ Тл, что дает минимум магнетосопротивления при экстраполяции к $\mathrm{H}=0$ $(\mathrm{n}=0)$. Это соответствует сдвигу на $\pi$ по сравнению с фазой осцилляций Аронова-Бома в случае слабой локализации, когда наблюдается максимум магнетосопротивления при $\mathrm{H} \rightarrow$ \pm 0 . Отметим, что анализ фазы осцилляций Шубникова-де Гааза образца №3, проведенный по схеме [13], дает фазу этих осцилляций, близкую к нулю (рис. 4), что характерно для дираковских фермионов [13].

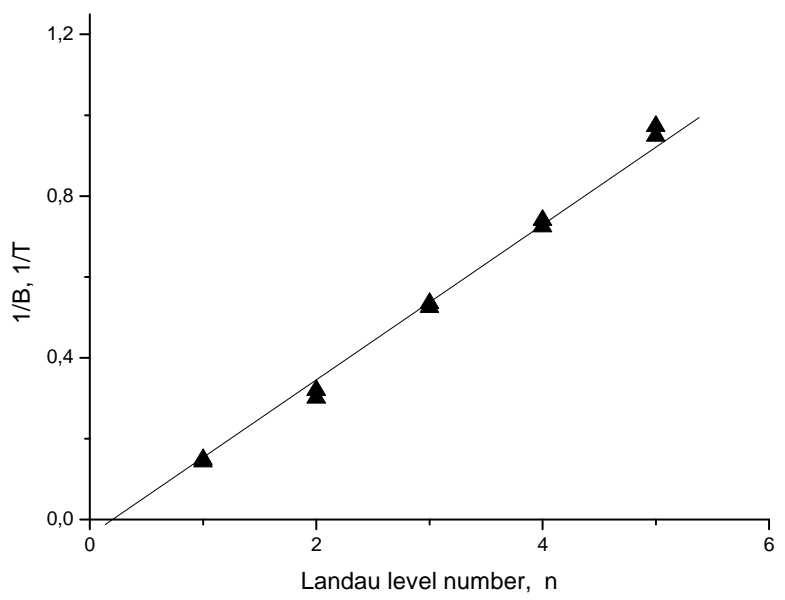

Рис. 4. Анализ фазы осцилляций магнетосопротивления Шубникова-де Гааза образца №3, полученный экстраполяций обратного поля, соответствующего минимуму осциллирующей части магнетосопротивления от номера соответствующего уровня Ландау n [13].

Другим качественным результатом эксперимента является большая амплитуда осцилляций, составляющая 10\% от неосциллирующего фона, что указывает на фазовую корреляцию большого числа носителей, локализованных на разных дефектах. Это возможно только в условиях, когда длина сбоя фазовой когерентности $l_{\varphi}$ носителей существенно превышает среднее расстояние между КД, составляющее при концентрации $10^{9}$ деф/см² 0,3 мкм. Вместе с тем, механизм этой синхронизации пока остается непонятным. Эффект Ааронова-Бома наблюдался на тонких кристаллах $\mathrm{NbSe}_{3}$ с КД в режиме скольжения волны зарядовой плотности (ВЗП) [14]. В этих экспериментах синхронизация эффекта на большом массиве КД связывалась с большой длиной фазовой корреляции движущейся ВЗП.

Отметим еще одно обстоятельство. Положение по Н минимумов крупномасштабных осцилляций образца №3 коррелирует с положением минимумов осцилляций Шубникова де Газа. В двумерных системах обычно минимуму магнетосопротивления соответствует плато холловского сопротивления с фиксированным фактором запонения $v, v=\mathrm{H}_{0} / \mathrm{H}$. C ростом Н минимум последней осцилляции магнетосопротивления Шубникова-де Гааза находится в области $\mathrm{H}=\mathrm{H}_{0}=7-7,5$ Тл, и соответствует $v=1$. Минимумы дальнейших, крупномасштабных осцилляций - периодические по полю с периодом 7.5 Тл. Учитывая, что $\mathrm{H}=\mathrm{H}_{0} / v$, им можно поставить в соответствие $v=1 / 2$ (15 Тл), 1/3 (22,5 Тл), 1/4 (30 Тл). Указанная корреляция прослеживается на рис. 5 , где соответствующие минимумам числа заполнения отложены, как функция $1 /$ Н. Точки при $v>1$ и $v<1$ ложатся на одну прямую. Это наблюдение показывает, что обнаруженные периодические по полю осцилляции магнетосопротивления могут и не быть связаны напрямую с эффектом Ааронова-Бома, а, 
возможно, определяться появлением новых состояний, порождаемых наличием КД, например, краевых.

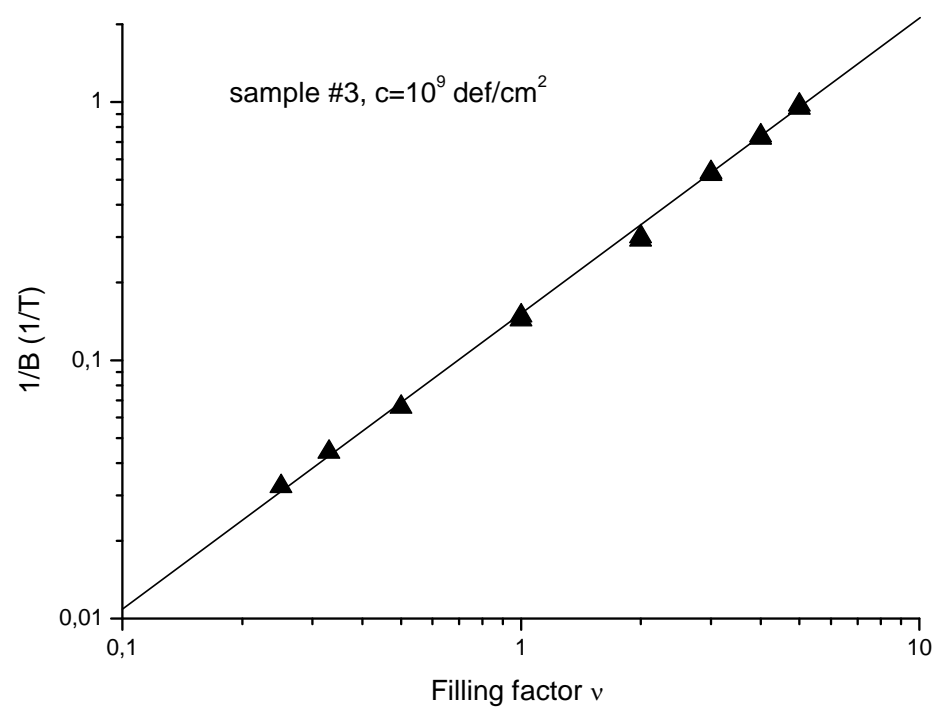

Рис. 5. Зависимость обратного поля, соответствующего минимумам осциллирующего магнетосопротивления от фактора заполнения $v$ образца №3 при Т=1.2 К.

Дальнейшее развитие эксперимента связывается с более точным определением размеров КД с использованием просвечивающей электронной микроскопии высокого разрешения. Мы также планируем продолжить эксперименты на более тонких кристаллах, биграфене и графене с различным диаметром КД и в более сильных, импульсных магнитных полях, а также дополнить измерения $\rho_{\mathrm{xx}}(\mathrm{H})$ измерениями $\rho_{\mathrm{xy}}(\mathrm{H})$.

Авторы признательны В.А. Волкову за плодотворные обсуждения результатов работы. Работа была поддержана грантами РФФИ № 08-02-01093а, № 06-02-72551, грантом ANR-07-BLAN-0136, Европейской ассоциированной лабораторией (LEA) между ИРЭ PAН и Институтом Неля CNRS, программами РAН «Сильно коррелированные электроны в твердых телах и структурах”, и “Физика новых материалов и структур».

1. K.S. Novoselov et al., Nature, 438, 197 (2005).

2. M. Orlita et al. Phys. Rev. Lett. 100, 136403 (2008).

3. G. Li and E. Andrei, Nature Phys. 3, 623 (2007).

4. Yu.I. Latyshev et al., Journal of Physics: Conference Series 129, 012032 (2008).

5. M.I. Katsnelson, K.S. Novoselov, A.K. Geim, Nature Physics, 2, 620 (2006).

6. Ю.Е. Лозовик, С.П. Меркулов, А.А. Соколик, УФН, 178, 757 (2008).

7. S. Russo et al. Phys. Rev. B 77, 085413 (2008).

8. M. Orlita et al., cond-mat:: 0805.0553v1.

9. J.V. Scneider, M. Orlita, M. Potemski, and D.K. Maude, Phys. Rev. Lett., 102, 166403 (2009).

10. H. Ochimizu et al., Phys. Rev. B 46, 1986 (1992).

11. Y. Kopelevich, B. Raqet, M. Goiran et al., cond-mat.: 0903.2369.

12. V.A. Skuratov et al. Nucl. Instr. and Meth. B, 203, 136 (2003).

13. I.A. Lukyanchuk, Y. Kopelevich, Phys. Rev. Lett., 97, 256801 (2006).

14. Yu. I. Latyshev, O. Laborde, P. Monceau, and S. Klaumünzer, Phys. Rev. Lett., 78, 919

(1997). 